\title{
The relationship between psychological distress and bullying victimisation among school-going adolescents in Ghana: a cross-sectional study
}

\author{
Diane Korkor Arhin ${ }^{1}$, Kwaku Oppong Asante ${ }^{1,2,3^{*}} \mathbb{0}$, Nuworza Kugbey ${ }^{3,4}$ and Mabel Oti-Boadi ${ }^{5}$
}

\begin{abstract}
Objective: One of the pervasive problems in schools that has adverse implication on the wellbeing of students, is bullying victimisation. However, within sub-Saharan Africa, only few studies have examined how psychological distress influences bullying victimisation. The purpose of this study was to explore the association between bullying victimization and psychological distress among school going adolescents in Ghana.

Results: One hundred and ninety-eight (198) male and female students completed a series of self-report questionnaires measuring psychological distress (anxiety, stress and depression) and bullying victimisation in a cross-sectional survey. Pearson product-moment correlation coefficient and standard multiple regression were used to analyse the data. Findings revealed that bullying victimisation was positively associated with all the domains of psychological distress: depression $(r=0.35, p<0.001)$, anxiety $(r=0.30, p<0.001)$ and stress $(r=0.35, p<0.001)$. Further analysis found depression to be the only significant predictor of bullying victimisation $(\beta=0.20 ; t=2.01 ; p<0.05)$. Taking into consideration that bullying victimization is a major problem among adolescents in schools, anti-bullying programmes should be implemented as this may promote improved wellbeing of school-going adolescents in Ghana.
\end{abstract}

Keywords: School-going adolescents, Bullying, Psychological health, Health promotion, Ghana

\section{Introduction}

One of the pervasive problems in schools that has adverse implication on the wellbeing of students, is bullying. The psychological implications of bullying have been found to be more problematic with children found at the early adolescence stage [1]. This could be alarming since growth at this stage of adolescence is very key in building a healthy personality in adulthood. Within the literature, depression was found to be positively correlated to bullying victimization $[2,3]$ but negatively correlated to bullying perpetration $[1,4]$.

Though the concept of bullying has received quite a number of attention in the developed countries [3,

\footnotetext{
*Correspondence: koppongasante@ug.edu.gh; kwappong@gmail.com

1 Department of Psychology, School of Social Sciences, University of Ghana, P. O. Box LG 84, Legon, Accra, Ghana

Full list of author information is available at the end of the article
}

5-7], only few studies have explored bullying victimisation among school going adolescents within Africa. Anecdotal evidence however shows a significant increase in the prevalence of bullying among school children and adolescents in Africa with a prevalence rates of $21-61 \%$ in South Africa [8], 78\% in Malawi [9] and 59\% in Ghana [1].

Literature has shown that victims of bullying experience poorer social, emotional, and physical health outcomes including signs of depression, suicidal ideation and loneliness $[1,9]$. It has also been shown that social isolation and loneliness are associated with bullying victimisation $[8,10]$. Recent systematic reviews have established a strong causal associations between bullying victimization and mental health problems such as depression, anxiety, poor general health and suicidal ideation and behaviours [11]. It has also been established that bullying victimisation is independently associated with significantly higher 
levels of psychological distress and reduced levels of emotional wellbeing [12]. Due to the dearth of research on bullying victimisation in developing countries, there remains a considerable gap in research that needs to be filled to provide an understanding into the scope and nature of the psychological effects of bullying victimisation as the findings in western countries may not necessarily be applied to the Ghanaian context. It has also been suggested that context and culture influences different patterns of victimization experiences among adolescents and young adults [13]. With no anti-bullying policy in place within Ghanaian schools in addition to the high prevalence of bullying reported among adolescents [1], there is therefore the need for a contextually relevant study to explore the various psychological distress that may be associated with bullying victimisation. The purpose of this study is to examine the relationship between psychological distress and bullying victimisation among school-going adolescents in Ghana. The findings of this study could help in the developing proactive anti-bullying intervention programmes for schools.

\section{Main text \\ Methods \\ Study sample}

A total of 198 students from three (3) junior high schools from the Ledzekuku Krowor Municipal Assembly (LEKMA) in the Greater Accra Region of Ghana were conveniently sampled to participate in the study. Participants age ranges from 11 to 19 years with an average age of 13.7 years $(S D=1.44)$. Over half of the sample $(53.5 \%)$ were females with the remaining being males. Approximately $39.3 \%(n=78)$ of the students were in first year, $34.8 \%(\mathrm{n}=69)$ in second year and the remaining $25.8 \%$ $(\mathrm{n}=51)$ third year students.

\section{Study design and protocol}

A cross-sectional analysis carried out over 3 months (March-May 2018) where students were approached, and the purpose of the study explained. Those who agreed to participate signed a consent form. They were handed a questionnaire to fill out and return to the researcher. It took approximately $20 \mathrm{~min}$ to complete the questionnaire and was collected on the same day. Participants did not receive any form of compensation for their participation.

\section{Study instrument}

Two instruments were used in this study. These were the Depression, Anxiety and Stress Scale-21 (DASS-21) [14] and the bullying victimisation scale. The DASS-21 is a well-structured questionnaire consisting of 3 subscales of 7 items each measuring depression, anxiety and stress. The depression subscale assessed the presence of self-blame, pessimism and loss of enjoyment. The anxiety subscale assessed state of persistent apprehension and worry, accompanied by physical symptoms of sympathetic activation. The stress subscale assessed the state of over-arousal, tenseness and the inability to relax. The internal consistencies of the three subscales were 0.71 , 0.79 and 0.81 for depression, stress and anxiety respectively. Items on the DASS-21 are scored on a 3-point Likert scale ranging from $0=$ does not apply to me at all to $3=$ applies to me very much or most of the time. The reliability of the DASS-21 has been established within the Ghanaian context [15-17]. In this study the Cronbach alphas for depression, stress and anxiety were $0.77,0.82$ and 0.88 respectively. Since, the DASS-21 distinguishes between depression, anxiety and stress as distinct manifestations of psychological distress, the total of the subscales were used in this study.

Bullying victimisation was assessed using 6 items selected from previous research which assessed bullying victimization in the past 30 days $[18,19]$. The response options are rated on a 5-point Likert-type scale ranging from 0 (never) to 4 (several times a week) with higher scores on the bullying victimization shows elevated levels of victimization. Some of the items on the bullying victimization are: "I am called names, made fun of, or teased in a hurtful way" and "I was forced or threatened to do things I did not want to". In this study the Cronbach alphas for bullying victimization was 0.72 .

\section{Statistical analysis}

The Statistical Package for Social Sciences (SPSS) version 24.00 was used to analyse the data. Pearson's correlation analysis was used to test the relationships among the study variables. A standard multiple regression analysis was conducted to identify which of the psychological distress dimension best predicts bullying victimisation. In the regression analysis, only variables that had significant correlation coefficients with the criterion variable were entered into the regression models. All statistical tests were performed using two-tailed examination, and a $p$ value of 0.05 or less was considered statistically significant.

\section{Results \\ Relationship between domains of psychological distress and bullying victimisation}

Our results in Table 1 showed that bullying victimisation was positively associated with depression $(r=0.35$, $p<0.001)$, anxiety $(r=0.30, p<0.001)$ and stress $(r=0.35$, $p<0.001)$. These results suggest that higher levels of stress, anxiety and depression are associated with a corresponding elevated level of bullying victimisation among school-going adolescents. 
Table 1 Correlation between psychological distress and bullying victimisation

\begin{tabular}{|c|c|c|c|c|c|c|}
\hline Variables & Mean & $S D$ & 1 & 2 & 3 & 4 \\
\hline 1. Bullying victimisation & 4.80 & 1.80 & 1 & $0.35^{* * *}$ & $0.30^{* * *}$ & $0.35^{* * *}$ \\
\hline 2. Stress & 5.99 & 2.02 & - & 1 & $0.69^{* * *}$ & $0.65^{* * *}$ \\
\hline 3. Anxiety & 6.06 & 2.39 & - & - & 1 & $0.62^{* * *}$ \\
\hline 4. Depression & 6.01 & 2.11 & - & - & - & 1 \\
\hline
\end{tabular}

*** $p<0.001$

Table 2 Multiple regression analysis of the predictors of bullying victimisation

\begin{tabular}{llllll}
\hline Model & $\boldsymbol{B}$ & $\boldsymbol{S E} \boldsymbol{B}$ & $\boldsymbol{\beta}$ & $\boldsymbol{t}$ & $\boldsymbol{p}$ values \\
\hline Depression & 0.18 & 0.09 & 0.20 & 2.01 & $0.046^{*}$ \\
Stress & 0.12 & 0.11 & 0.12 & 1.10 & 0.272 \\
Anxiety & 0.10 & 0.10 & 0.10 & 0.98 & 0.330 \\
$R^{2}$ & & 0.337 & & & \\
$F$ & & $9.386^{* * *}$ & & & \\
\hline
\end{tabular}

$B$ unstandardised beta, SEM the standard error of the unstandardised beta, $\beta$ standardised beta

${ }^{*} p<0.05 ;{ }^{* * *} p<0.001$, two-tailed

\section{Predictors of bullying victimisation}

Standard multiple regressions were conducted to determine which variables significantly predicted bullying victimisations among school-going adolescents. The results, as presented in Table 2, showed that the regression model was significant $(F=9.40, p<0.001)$; and accounted for $37.7 \%$ of the variance in bullying victimisation $\left(R^{2}=0.337\right)$. Further analysis revealed depression to be the only significant predictor of bullying victimisation $(\beta=0.20 ; t=2.01 ; p<0.05)$. The psychological distress dimension of stress and anxiety did not significantly predict bullying victimisation.

\section{Discussion}

Findings from the study showed significant positive correlation between domains of psychological distress (depression, anxiety and stress) and bullying victimatization among school going adolescents in Ghana which suggest that elevated levels of psychological distress may pose as significant risk factors for bullying victimization $[3,20,21]$. The findings are not surprising as mental health problems among school going-adolescents predispose them to various risky behaviours including unsafe sexual behaviours and substance use [22, 23]. The symptoms of psychological distress could inhibit students' ability to resist bullying as perpetrators of bullying victimization may target their colleagues who are unable to fight back.
We further examined the individual contributions of the three psychological distress domains and found depression to be the only significant predictor of bullying victimization among the students. This means that adolescent students who experience elevated depression levels are at higher risks of being bullied compared to those with lower levels of depression. Since depression as a mental health problem is characterized by sadness, isolation, loss of interest in previously enjoyed activities, lethargy and suicidal behaviours, students with depressive symptoms may appear as weak and unable to resist acts of aggression from their peers. This therefore makes depressed students targets of bullying. This finding is consistent with previous works among students which found psychological distress, social isolation and loneliness to be significantly associated with bullying victimization [8-13].

The implication of these findings are that prevalence of mental health problems among school going adolescents could serve as a significant risk factor for bullying victimization and creates the need for regular screening of in-school adolescents for common mental health problems. There is also the need for mental health promotion activities among adolescents in schools to ensure prevention, identification and early treatment to reduce their adverse impacts on academic and other wellbeing outcomes. Violence prevention policies and measures should be put in place in our schools to deal with perpetrators of bullying.

\section{Conclusion}

This study demonstrated significant positive relationships among domains of psychological distress and bullying victimization among school-going adolescents which suggest the need for conscious efforts in identifying students at risk for bullying victimization especially those experiencing some form of psychological distress. Depression was the main contributing factor to the experience of more bullying victimization and as such intervention strategies should be tailored to this direction. There is the need for further studies to 
examine the mechanisms underlying the link between psychological distress and bullying victimization among school-going adolescents.

\section{Limitations}

Our study is limited by the relatively small sample size and the use of a cross-sectional design as no causal inferences could be drawn between the variables used in this study. Another limitation was the convenient sampling which makes generalization difficult. A more representative sample could increase the generalizability of the results. There was also the potential of responses to be affected by social desirability as the study relied on self-report measures. However, previous studies mainly focused on the effects of bullying victimization of the health and wellbeing of adolescents whereas this study identified risk factors which may serve as a basis for future research on the risk factors for bullying victimization.

\section{Abbreviations}

DASS: Depression, Anxiety and Stress Scale; LEKMA: Ledzekuku Krowor Municipal Assembly; SPSS: Statistical Package for Social Sciences; SEM: the standard error of the unstandardised beta.

\section{Acknowledgements}

The authors wish to express our profound gratitude to all the school children who volunteered to participate in the study. We would also like to acknowledge the school administrators who gave us approval to use their students as our population.

\section{Authors' contributions}

DKA designed the study and collected data. KOA was involved in design, data analysis and drafting of the manuscript. NK and MOB were involved in data analysis and drafting of the manuscript. KOA reviewed the manuscript for key intellectual content. All authors read and approved the final manuscript.

\section{Funding}

No funding received for this study.

\section{Availability of data and materials}

The datasets used and/or analysed during the current study are available from the lead and corresponding authors on reasonable request.

\section{Ethics approval and consent to participate}

Ethical protocols for the study was approved by the Research and Ethics Committee of the Department of Psychology, University of Ghana, Accra, Ghana. All procedures performed in studies involving human participants were in accordance with the ethical standards of the institutional and/or national research committee and with the 1964 Helsinki declaration and its later amendments or comparable ethical standards. Written informed consent was obtained from students aged 18 years and above while parental consent was from students who were less than 18 years prior to their participation in the study. All other key ethical principles were followed.

\section{Consent for publication}

The signed informed and parental consents were informative to participants regarding the use of their data for academic purposes including publications in journals.

\section{Competing interests}

The authors declare that they do not have any conflict of interests.

\section{Author details}

${ }^{1}$ Department of Psychology, School of Social Sciences, University of Ghana, P. O. Box LG 84, Legon, Accra, Ghana. ${ }^{2}$ Institute for Psychosocial Research on Child and Adolescent Wellbeing, Accra, Ghana. ${ }^{3}$ Discipline of Psychology, University of KwaZulu-Natal, Durban, South Africa. ${ }^{4}$ Department of Family and Community Health, University of Health and Allied Sciences, Hohoe Campus, Volta Region, Ghana. ${ }^{5}$ General Studies Department, Ghana Technology University College, Accra, Ghana.

Received: 13 March 2019 Accepted: 3 May 2019

Published online: 10 May 2019

\section{References}

1. Owusu A, Hart P, Oliver B, Kang M. The association between bullying and psychological health among senior high school students in Ghana, West Africa. J Sch Health. 2011:81:231-8.

2. Bradshaw CP, Waasdorp TE, Johnson SL. Overlapping verbal, relational, physical, and electronic forms of bullying in adolescence: influence of school context. J Clin Child Adolesc Psychol. 2015;44:494-508.

3. Fullchange A, Furlong MJ. An exploration of effects of bullying victimization from a complete mental health perspective. SAGE Open. 2016:6:1-12.

4. Kaltiala-Heino R, Fröjd S. Correlation between bullying and clinical depression in adolescent patients. Adolesc Health Med Ther. 2011;2:37-44

5. Albdour M, Krouse HJ. Bullying and victimization among African American adolescents: a literature review. J Child Adolesc Psychiatr Nurs. 2014;27:68-82.

6. Cho Y, Chung OB. A mediated moderation model of conformative peer bullying. J Child Fam Stud. 2012;21:520-9.

7. Maniglio R. Bullying and other forms of peer victimization in adolescence and alcohol use. Trauma Violence Abuse. 2017;18:457-73.

8. Steyn GM, Singh GD. Managing bullying in South African secondary schools: a case study. Int J Educ Manag. 2018;32:1029-40.

9. Kim YK, Okumu M, Small E, Nikolova SP, Mengo C. The association between school bullying victimization and substance use among adolescents in Malawi: the mediating effect of loneliness. Int J Adolesc Med Health. 2018. https://doi.org/10.1515/ijamh-2017-0229.

10. Acquah EO, Topalli P, Wilson ML, Junttila N, Niemi PM. Adolescent loneliness and social anxiety as predictors of bullying victimisation. Int J Adolesc Youth. 2016;21:320-31.

11. Moore SE, Norman RE, Suetani S, Thomas HJ, Sly PD, Scott JG. Consequences of bullying victimization in childhood and adolescence: a systematic review and meta-analysis. World J Psychiatry. 2017:7:60-76.

12. Thomas HJ, Chan GC, Scott JG, Connor JP, Kelly AB, Williams J. Association of different forms of bullying victimisation with adolescents' psychological distress and reduced emotional wellbeing. Aust N Z J Psychiatry. 2016:50:371-9.

13. Nguyen AJ, Bradshaw C, Townsend L, Bass J. Prevalence and correlates of bullying victimization in four low-resource countries. J Interpers Violence. 2017;1:13. https://doi.org/10.1177/0886260517709799.

14. Lovibond SH, Lovibond PF. Manual for the depression anxiety stress scales. 2nd ed. Sydney: Psychology Foundation of Australia; 1995.

15. Oppong Asante K. Social support and the psychological wellbeing of people living with HIV/AIDS in Ghana. Afr J Psychiatry. 2012;15(5):340-5.

16. Kretchy IA, Owusu-Daaku FT, Danquah SA. Mental health in hypertension: assessing symptoms of anxiety, depression and stress on anti-hypertensive medication adherence. Int J Ment Health Syst. 2014;8(1):25.

17. Opoku-Boateng YN, Kretchy IA, Aryeetey GC, Dwomoh D, Decker S, Agyemang SA, Tozan Y, Aikins M, Nonvignon J. Economic cost and quality of life of family caregivers of schizophrenic patients attending psychiatric hospitals in Ghana. BMC Health Serv Res. 2017;17(2):697.

18. Olweus D. Bullying at school: what we know and what we can do. Malden: Blackwell Publishing; 1993.

19. Centers for Disease Control and Prevention (CDC). Global Student Health Survey (GSHS). Geneva: World Health Organization; 2013.

20. Cook CR, Williams KR, Guerra NG, Kim TE, Sadek S. Predictors of bullying and victimization in childhood and adolescence: a meta-analytic investigation. Sch Psychol Q. 2010;25:65-83. 
21. Kowalski RM, Limber SP. Psychological, physical, and academic correlates of cyberbullying and traditional bullying. J Adolesc Health. 2013;53:S13-20.

22. Kugbey N, Ayanore MA, Amu H, Oppong Asante K, Adam A. International note: analysis of risk and protective factors for risky sexual behaviours among school-aged adolescents. J Adolesc. 2018;68:66-9.

23. Oppong Asante K, Kugbey N. Alcohol use by school-going adolescents in Ghana: prevalence and correlates. Ment Health Prev. 2019;13:75-81.

\section{Publisher's Note}

Springer Nature remains neutral with regard to jurisdictional claims in published maps and institutional affiliations.
Ready to submit your research? Choose BMC and benefit from:

- fast, convenient online submission

- thorough peer review by experienced researchers in your field

- rapid publication on acceptance

- support for research data, including large and complex data types

- gold Open Access which fosters wider collaboration and increased citations

- maximum visibility for your research: over 100M website views per year

At BMC, research is always in progress.

Learn more biomedcentral.com/submissions 